\title{
A Lagrangian Relaxation-based Heuristic to Solve Large Extended Graph Partitioning Problems
}

\author{
Oliver G. Czibula ${ }^{1,2}$, Hanyu $\mathrm{Gu}^{2}$, and Yakov Zinder ${ }^{2}$ \\ 1 Ausgrid, 570 George Street Sydney NSW 2000, Australia \\ 2 School of Mathematical and Physical Sciences, University of Technology Sydney \\ 15 Broadway Ultimo NSW 2007, Australia
}

\begin{abstract}
The paper is concerned with the planning of training sessions in large organisations requiring periodic retraining of their staff. The allocation of students must take into account student preferences as well as the desired composition of study groups. The paper presents a bicriteria Quadratic Multiple Knapsack formulation of the considered practical problem, and a novel solution procedure based on Lagrangian relaxation. The paper presents the results of computational experiments aimed at testing the optimisation procedure on real world data originating from Australia's largest electricity distributor. Results are compared and validated against a Genetic Algorithm based matheuristic.
\end{abstract}

\section{Introduction}

This paper is concerned with the optimisation of class formation at large organisations, typically with thousands of workers of different types, that require periodic retraining of their staff. Finding good solutions to this problem is important as it allows more effective training sessions to be provided.

This research is motivated by the problem of providing training to workers at Ausgrid, Australia's largest electricity distributor. Due to the multitude of hazards that exist when working with high voltages at heights or in confined spaces, Ausgrid is required by Australian law to deliver regular safety, technical, and professional training to all its employees who work on or near the electricity network. Ausgrid provides regular training to thousands of employees, contractors, and third parties. Many of these people have very different learning outcomes from courses, different learning styles, different levels of education or English proficiency, and different levels of technical proficiency for certain tasks.

Consider an example where field workers and upper management are undertaking a particular course: while the core material would remain the same for both groups, if they are taught in separate classes the trainers can take the opportunity to better tailor the delivery to the specific needs of their group, allowing for more productive training sessions. It is often not possible to run segregated classes due to the scarcity of training resources and the associated cost of delivering additional classes, therefore some blending of different student 
types is often necessary. Ideally, differing student types should be combined into a single class only when they have a high compatibility with one another.

In similar problems where students are preferentially assigned to classes, it is customary to give each student-class pair a weight (or cost). Most of the training provided by Ausgrid has a limited period in which it is valid, and therefore courses should be periodically retrained. Workers are only permitted to work in roles for which they have up-to-date training. The date at which a worker's training expires is considered their due date for that course. If there are a number of scheduled classes for a given course that a worker requires, we can designate a cost of assigning the worker to any one of those classes: classes that run on or before their due date have low or zero cost, while classes that run after the due date have a high cost that increases the later the class is scheduled.

The considered problem has a bi-criteria objective: we wish to find an assignment of students to classes that minimises incompatibility between student types within classes, and that minimises the assignment cost of individual students to classes. Each class has a minimum and maximum number of students it can hold, and of student types it can be assigned.

Johnson et al. [10] discuss the problem of partitioning the vertices of a graph $G(V, E)$ with nonnegative weights $w_{v}, v \in V$ and $\operatorname{costs} c_{e}, e \in E$, into $K$ disjoint clusters partitioning $V$, such that the sum of the weights in each cluster is bounded between $w_{\min }$ and $w_{\max }$ and the sum of the costs within each cluster is minimised. This problem is known as the graph partitioning problem, and is known to be NP-hard [4], [7]. Johnson et al. proposed a column generation approach, and tested the approach on graphs with between 30 and 61 nodes, and between 47 and 187 edges. For the 12 test cases the authors considered, their proposed approach provided integer solutions for all but two, and for those, solutions obtained by a branch-and-bound scheme were very close to the fractional solutions provided by column generation.

The problem considered in this paper can be modelled as a generalised graph partitioning problem. Each student $j$ is represented by a vertex $v_{j} \in V$; the preference between students $i$ and $j$ is represented by an undirected edge $e=\left(v_{i}, v_{j}\right) \in E$ with edge cost $c_{e}$. The problem is to find a partition $\Gamma=$ $\left\{W_{1}, W_{2}, \ldots, W_{N}\right\}$ of $V$ that solves

$$
\begin{gathered}
\text { Minimise: } \quad \alpha \sum_{i=1}^{N} \sum_{e \in E\left(W_{i}\right)} c_{e}+\beta \sum_{i=1}^{N} \sum_{v_{k} \in W_{i}} w_{v_{k}}^{i} \\
\text { Subject To: } \quad w_{\min } \leq\left|W_{i}\right| \leq w_{\max } \quad i=1, \ldots, k
\end{gathered}
$$

where $N$ is the number of classes, $W_{i}$ is the set of students assigned to class $i$, $E\left(W_{i}\right)=\left\{\left(v_{k}, v_{l}\right) \mid v_{k} \in W_{i}, v_{l} \in W_{i}\right\}, w_{v_{k}}^{i}$ is cost of assigning student $k$ to class $i, \alpha$ and $\beta$ are weights for the assigning cost and preference cost respectively. A special case of the problem considered in this paper, in which $w_{v_{k}}^{1}=w_{v_{k}}^{2}=\ldots=$ $w_{v_{k}}^{N}$, is equivalent to the graph partitioning problem. Therefore our problem is also NP-hard. 
Chopra and Rao [3] discuss several forms of the graph partitioning problem as well as IP models for each. The authors do not assume a complete graph, allowing them to take advantage of the graph structure when clustering. They also discuss several valid inequalities and facet-defining inequalities for the GPP.

The considered problem can also be modelled as an extended Quadratic Multiple Knapsack Problem (QMKP) with additional constraints. The QMKP is a generalization and combination of the well-known multiple knapsack problem and the quadratic knapsack problem. The QMKP received little attention in the literature until recently, and most solution approaches are based on metaheuristics [2], [6], [9]. The main contributions of this paper include: (i) formulation of the considered practical problem as an extended bicriteria QMKP. (ii) design of a Lagrangian relaxation (LR) based, fast heuristic capable of solving large, real-world instances.

Caprara et al. [1] discuss an LR-based approach to solving the QMKP exactly, whereby a tighter upper bound (for their maximisation objective) is computed using the subgradient method in linear expected time. The presented approach is able to solve instances with up to 400 binary variables exactly. The authors note that the presented approach can also be used to solve Max Clique problems almost as fast as with the cutting plane approaches available at the time.

Julstrom [11] discusses greedy, genetic, and greedy genetic algorithms for the QMKP. The author presents two greedy heuristics that build solutions by choosing objects according to their value densities, and two genetic algorithm (GA) heuristics. One GA is a standard implementation, whereas the other is extended with greedy techniques that probabilistically favour objects of high value density. The four algorithms are tested on 20 problem instances, and the extended GA is reported to perform best on all but one test case.

The remainder of this paper is organised as follows: Section 2 introduces a quadratic programming formulation and its linearisation for the considered problem; Section 3 introduces a Lagrangian relaxation formulation for the quadratic programming model; Section 4 discusses the proposed LR-based heuristic technique; Section 5 presents a GA matheuristic for the problem; Section 6 presents the computational results of the proposed heuristic on a number of industryinspired test cases; and Section 7 discusses our conclusions and outlines possibilities for future research.

\section{Quadratic Programming Formulation}

Let $\mathcal{N}=\{1, \cdots, N\}, \mathcal{M}=\{1, \cdots, M\}$, and $\mathcal{K}=\{1, \cdots, K\}$ be the set of classes available, the set of students to be assigned, and the set of student types respectively. Denote the cost of assigning students $j \in \mathcal{M}$ to class $i \in \mathcal{N}$ by $c_{i, j}$, the cost of pairing student types $k \in \mathcal{K}$ and $l \in \mathcal{K}$ together in the same class by $b_{k, l}$. Each student has exactly one type. The set of students who are of type $k$ is represented by $T_{k}, k \in \mathcal{K}$. Each student must be assigned to exactly one class, but not all classes need to be run. Each class $i \in \mathcal{N}$ that is run must contain at least $a_{i}$ and at most $b_{i}$ students, and at least $p_{i}$ and at most $q_{i}$ student types. 
Students or student types cannot be assigned to any class that is not run. In this problem, we assume there are more scheduled classes than needed, i.e. a feasible solution always exists. The following Quadratic Program describes the problem:

$$
\begin{array}{rl}
\text { (QP) Minimise: } \alpha \sum_{i=1}^{N} \sum_{k=1}^{K} \sum_{l=1}^{K} b_{k, l} Y_{i, k} Y_{i, l}+\beta \sum_{i=1}^{N} \sum_{j=1}^{M} c_{i, j} X_{i, j} \\
\text { Subject To: } \sum_{i=1}^{N} X_{i, j}=1 \quad j=1, \ldots, M \\
a_{i} Z_{i} \leq \sum_{j=1}^{M} X_{i, j} \leq b_{i} Z_{i} \quad i=1, \ldots, N \\
p_{i} Z_{i} \leq \sum_{k=1}^{K} Y_{i, k} \leq q_{i} Z_{i} & i=1, \ldots, N \\
X_{i, j} \leq Y_{i, k} & i=1, \ldots, N ; k=1, \ldots, K ; j \in T_{k} \\
X_{i, j} \in\{0,1\} & i=1, \ldots, N ; j=1, \ldots, M \\
Y_{i, k} \in\{0,1\} & i=1, \ldots, N ; k=1, \ldots, K \\
Z_{i} \in\{0,1\} & i=1, \ldots, N
\end{array}
$$

where the binary variable $X_{i, j}$ is defined to be 1 if student $j$ is assigned to class $i$, or 0 otherwise; the binary variable $Y_{i, k}$ is defined to be 1 if student type $k$ is assigned to class $i$, or 0 otherwise; The binary variable $Z_{i}$ is defined to be 1 if class $i$ is run, or 0 otherwise.

In the objective function (3), the quadratic term represents the cost of pairing student types together, and the linear term represents the cost of assigning students to classes, weighted by coefficients $\alpha$ and $\beta$, respectively.

The constraints (4) express the requirement that each student be assigned to exactly one class. The constraints (5) and (6) express the requirement that each running class has between $a_{i}$ and $b_{i}$ students and between $p_{i}$ and $q_{i}$ student types, respectively, if the class is run, or zero otherwise. The constraints (7) express the requirement that a student may only be assigned to a class if that student's type has also been assigned to that class.

It is possible to linearise the quadratic term in (3) by introducing $\hat{Y}_{i, k, l}=$ $Y_{i, k} Y_{i, l}$ together with constraints:

$$
\begin{aligned}
& \hat{Y}_{i, k, l} \leq Y_{i, k} \quad i=1, \ldots, N ; k=1, \ldots, K ; l=1, \ldots, K \\
& \hat{Y}_{i, k, l} \leq Y_{i, l} \quad i=1, \ldots, N ; k=1, \ldots, K ; l=1, \ldots, K \\
& \hat{Y}_{i, k, l} \geq Y_{i, k}+Y_{i, l}-1 \quad i=1, \ldots, N ; k=1, \ldots, K ; l=1, \ldots, K
\end{aligned}
$$

to give the linearised model: 
(LQP) Minimise: $\alpha \sum_{i=1}^{N} \sum_{k=1}^{K} \sum_{l=1}^{K} b_{k, l} \hat{Y}_{i, k, l}+\beta \sum_{i=1}^{N} \sum_{j=1}^{M} c_{i, j} X_{i, j}$

Subject To: $(4)-(13)$

$$
\hat{Y}_{i, k, l} \in\{0,1\} \quad i=1, \ldots, N ; k=1, \ldots, K ; l=1, \ldots, K
$$

The (QP) model has $N(K+M+1)$ variables and $M+2 N+M N K$ constraints, whereas the (LQP) model has $N\left(K+M^{2}+M+1\right)$ variables and $M+2 N+$ $M N K+3 N K^{2}$ constraints.

The time required to find an optimal solution to the (QP) and (LQP) models grows rapidly, where even some small test cases with just a few dozen students can take hours to solve. As the problem instances we hope to solve are significantly larger than this, we propose to use a heuristic approach.

\section{Lagrangian Relaxation}

To improve the convergence of the subgradient algorithm, the equalities (4) are first converted into inequalities:

$$
\sum_{i=1}^{N} X_{i, j} \geq 1 \quad j=1, \ldots, M
$$

By moving the constraints (4) into the objective function, we obtain the Lagrangian relaxation model:

(QR) Minimise: $\alpha \sum_{i=1}^{N} \sum_{k=1}^{K} \sum_{l=1}^{K} b_{k, l} Y_{i, k} Y_{i, l}+\beta \sum_{i=1}^{N} \sum_{j=1}^{M} c_{i, j} X_{i, j}+\sum_{j=1}^{M} \lambda_{j}\left(1-\sum_{i=1}^{N} X_{i, j}\right)$

$$
\text { Subject To: }(5)-(10)
$$

where $\lambda_{j} \geq 0, j=1, \ldots, M$, is the Lagrangian multiplier corresponding to the cost of not assigning student $j$ to any class. The quadratic term in (18) can be linearised in the same way as with (3).

In (QP), only constraints (4) couple together the $N$ sub-problems of assigning students to a particular class $i$. In (QR), these constraints are moved to the objective function, therefore it is possible to express (QR) as $N$ smaller, classspecific sub-problems $\left(\mathrm{QR}_{i}\right)$ :

$$
\left(\mathrm{QR}_{i}\right) \text { Minimise: } \alpha \sum_{k=1}^{K} \sum_{l=1}^{K} b_{k, l} Y_{i, k} Y_{i, l}+\beta \sum_{j=1}^{M} c_{i, j} X_{i, j}-\sum_{j=1}^{M} \lambda_{j} X_{i, j}
$$




$$
\begin{aligned}
& \text { Subject To: } \quad a_{i} Z_{i} \leq \sum_{j=1}^{M} X_{i, j} \leq b_{i} Z_{i} \\
& \qquad p_{i} Z_{i} \leq \sum_{k=1}^{K} Y_{i, k} \leq q_{i} Z_{i} \\
& X_{i, j} \leq Y_{i, k} \quad k=1, \ldots, K ; j \in T_{k} \\
& X_{i, j} \in\{0,1\} \quad j=1, \ldots, M \\
& Y_{i, k} \in\{0,1\} \quad k=1, \ldots, K \\
& Z_{i} \in\{0,1\}
\end{aligned}
$$

where the $N$ combined solutions to $\left(\mathrm{QR}_{i}\right)$ for $1 \leq i \leq N$ forms the solution to (QR).

Solving the Lagrangian relaxation $(\mathrm{QR})$ provides a lower bound to the optimal objective value of (QP). We wish to find the tightest possible lower bound for (QP) by solving the Lagrangian dual problem, the solution of which are the optimal Lagrangian multipliers $\lambda^{*}$ that give the largest objective value of (QR) [8], [5] . A typical way to numerically approximate $\lambda^{*}$ is by using the iterative subgradient algorithm.

In the subgradient algorithm, $\lambda$ is iteratively updated with the relationship

$$
\lambda^{k+1}=\lambda^{k}+\frac{s^{k} \cdot \epsilon_{k}\left(\eta^{*}-\eta^{k}\right)}{\left\|s^{k}\right\|^{2}}
$$

where $\lambda^{k}$ is the value of the Lagrangian multipliers $\lambda$ at the $k$ th iteration; $\eta^{*}$ is an upper bound of the optimal objective value of the Lagrangian dual problem, and $\eta^{k}$ is the objective value of $(\mathrm{QR})$ at the $k$ th iteration; and $\epsilon_{k}$ is a positive scaling factor, typically with the initial value of $\epsilon_{0}=2$ and halved whenever the objective value hasn't been improved in certain number of iterations; $s^{k}$ is a subgradient of the Lagrangian dual at iteration $k$ given by

$$
s_{j}^{k}=1-\sum_{i=1}^{N} X_{i, j}^{k} \quad j=1, \ldots, M
$$

where $X^{k}$ is from the solution of $(\mathrm{QR})$ at the $k$ th iteration.

\section{Lagrangian Heuristic}

In our proposed heuristic, we solve the Lagrangian dual using the subgradient algorithm. In doing so, we generate many solutions in this iterative process, and we record the following characteristics about them:

- The number of times class $i$ is running $\left(\xi_{i}\right)$, and the number of times it is $\operatorname{not}\left(\bar{\xi}_{i}\right)$.

- The number of times type $k$ is assigned to class $i\left(\phi_{i, k}\right)$, and the number of times it is not $\left(\bar{\phi}_{i, k}\right)$. 
- The number of times students $j_{1}$ and $j_{2}$ are assigned to the same class $\left(\psi_{j_{1}, j_{2}}\right)$, and the number of times they are not $\left(\bar{\psi}_{j_{1}, j_{2}}\right)$.

Based on these values, one or more assumptions are made about the final solution with some probability. The higher the value, the greater the probability the assumption will be made. Each value has a corresponding counter-value, such as the number of times class $i$ is running $\left(\xi_{i}\right)$, and the number of times class $i$ is not running $\left(\bar{\xi}_{i}\right)$. An assumption can be made according to the value/countervalue that has greater magnitude, for example if $\xi_{i}=7649$ and $\bar{\xi}_{i}=1008$ then, since $\xi_{i}>\bar{\xi}_{i}$, we would be making the assumption that class $i$ will be run, with some probability. The probability with which we make an assumption according to some value $v$ ( or counter-value $\bar{v}$ ) is $\frac{v}{v+\bar{v}}^{2}$ (or $\frac{\bar{v}}{v+\bar{v}}$ ), so if $\xi_{i}=7649$ and $\bar{\xi}_{i}=1008$ then with probability $\left(\frac{7649}{7649+1008}\right)^{2} \approx 0.7807$ we will make the assumption that class $i$ will run in the final solution.

Each kind of imposed assumption reduces the number of feasible solutions of the model being solved. In the case of the first kind of assumption, the $Z_{i}$ variable is fixed to 1 , if class $i$ is assumed running, or if class $i$ is assumed not running then the $Z_{i}$ and the related $X_{i, j}$ and $Y_{i, k}$ variables, $1 \leq j \leq M$ and $1 \leq k \leq K$, can be all fixed to zero. For the assumption that type $k$ is or is not assigned to class $i$, the constraints (7) can be omitted for $j \in T_{k}$, and all the related $X_{i, j}$ variables are also fixed to zero if type $k$ is assumed to be not assigned to class $i$. For the assumption that students $j_{1}$ and $j_{2}$ should be assigned together, we introduce the additional constraints $X_{i, j_{1}}=X_{i, j_{2}}$ for $1 \leq i \leq N$, and for the assumption that they should be assigned separately, we introduce the constraints $X_{i, j_{1}}+X_{i, j_{2}} \leq 1$ for $1 \leq i \leq N$.

Once an assumption is made, the model is updated and the Lagrangian dual is once again solved using the subgradient algorithm. With each new assumption the size of the model becomes smaller or the number of feasible solutions is reduced. Once the model is sufficiently small, the optimal solution, subject to the assumptions, can be found using a commercial solver.

The initial value of $\lambda=\lambda^{0}$ is likely to be quite distant from $\lambda^{*}$. It is expected that there would be an initial period of convergence from $\lambda^{0}$ towards the neighbourhood of $\lambda^{*}$, followed by a period of convergence within this neighbourhood. Since these early values of $\lambda^{k}$ are likely to produce fairly poor solutions, we treat this early period of convergence towards the neighbourhood of $\lambda^{*}$ as a "burn-in stage", and do not record these solutions.

To calculate an upper bound for the Lagrangian dual in (27), we relax constraints (7) from the (QP) model. The resulting capacitated assignment problem can be solved quickly, and a repair heuristic is used to make the solution feasible with respect to the original problem. The objective value of this feasible solution is used as $\eta^{*}$ in the updating rule (27) of the subgradient algorithm.

Our LR-based heuristic is described as follows:

\section{LR-based Heuristic:}

Step 1. Initialise an empty set of assumptions A.

Step 2. Construct the (QP) model, together with assumptions A. 
i. If the size of the model is sufficiently small to solve in practically acceptable time, attempt to solve it:

(a) If a solution with sufficiently small relative IP gap can found within a short time limit, terminate the algorithm returning the optimal solution to the (QP) subject to assumptions A.

(b) If no solution with sufficiently small relative IP gap can be found within a short time limit, continue to Step 3.

(c) If no feasible solution exists, determine which assumptions are causing the infeasibility, remove them, and continue to Step 3.

ii. If the size of the model is expected to be too large to solve in practically acceptable time, continue to Step 3.

Step 3. Run LD-Subroutine and retrieve all the values of $\xi, \bar{\xi}, \phi, \bar{\phi}, \psi$, and $\bar{\psi}$.

Step 4. For each of the value and counter-value pairs $\{v, \bar{v}\}$ obtained in Step 3, add an assumption to A with probability $\frac{v}{v+\bar{v}}^{2}\left(\right.$ or $\left.\frac{\bar{v}}{v+\bar{v}}^{2}\right)$ and then return to Step 2.

\section{LD-Subroutine:}

Step 1. Initialise the $\lambda$ vector to its starting value $\lambda^{0}, \epsilon_{0}:=2$, determine an estimate for $\eta^{*}$, initialise the iteration counter $k:=0$, and initialise all $\xi, \bar{\xi}$, $\phi, \bar{\phi}, \psi$, and $\bar{\psi}$ values to zero.

Step 2. Construct the $N\left(\mathrm{QR}_{i}\right)$ models according to the input data and the set of assumptions $\mathrm{A}$, for $1 \leq i \leq N$.

Step 3. Solve the $N\left(\mathrm{QR}_{i}\right)$ models, and update $\lambda$ according to (27).

Step 4. If $\epsilon_{k} \leq \frac{1}{2}$, update the values of $\xi, \bar{\xi}, \phi, \bar{\phi}, \psi$, and $\bar{\psi}$ according to observations about solution $X^{k}$.

Step 4. If the $\eta^{k}$ has not been improved in the last $10 \times N$ iterations, then $\epsilon_{k+1}:=\frac{1}{2} \epsilon_{k}$.

Step 5. If $\epsilon_{k}<0.1$ then terminate LD-Subroutine and return the values of $\xi, \bar{\xi}$, $\phi, \bar{\phi}, \psi$, and $\bar{\psi}$; otherwise set $k:=k+1$ and return to Step 2 .

\section{Genetic Algorithm Based Matheuristic}

Although Genetic Algorithms (GA) are often used in solving the QKP and QMKP [13], [14], [9], these publications on GA are not directly applicable to our problem due to the existence of lower bounds on class sizes. Moreover, the existence of lower and upper bounds on class sizes renders classical operations of crossover and mutation inefficient, i.e. both operations produce too many infeasible solutions. The computational experiments indicated that the common approach of introducing penalty for infeasibility does not improve the performance of the classical version of GA. These observations lead to the development of a matheuristic, presented in this paper, which is an amalgamation of GA and IP. This matheuristic was compared with the LR-based approach described above.

In the developed version of GA, feasible solutions in the initial population are be generated using a two-step procedure. First, we decide which classes will be run, and how many students will be in each class by solving a straightforward 
IP. For all test cases, including all cases where CPLEX failed to solve the original QP problem, this IP was solved in under a second. Next, we randomly assign students to classes according to the numbers obtained in the previous step.

The crossover operator, which addresses the challenge imposed by the existence of lower and upper bounds on class sizes, is defined as follows. As with conventional crossover operators, the designed crossover operates on two solutions, referred to as parents. The results of the designed crossover is a single solution, referred to as a child. As with the procedure that generates solutions for the initial population, the crossover operator involves two stages. First, an IP is solved that determines which classes should be run in the child, and the number of students in each class. In this IP, those classes that are run in both parents must run in the child, and those classes that are not run in either parent will not run in the child.

Next, students are assigned to classes in numbers specified by the IP. In this stage, first, students are assigned starting with students who are assigned to the same class in both parents. Each of these students is assigned to the that class in the child as well. The remaining students are assigned one at a time. If at least one class chosen for the student in the parents is available for this student in the child, then the student is assigned to one of these classes. If there are two such classes, the actual class is chosen at random. Students who were not allocated are then randomly allocated to the classes in the child solution according numbers specified by the IP.

\section{Computational results}

Using Ausgrid's training data as a template, we generated a series of random test cases $^{3}$. Each test case had between 100 and 500 students, with between 4 and 12 student types, and between 56 and 98 classes.

We used IBM ILOG CPLEX 12.5.0.0 64-bit on an Intel i7-4790K quad-core 4.00Ghz system with 16GB of RAM, running Windows 7 Professional. Our code was written in C\# 4.0, and interacted with CPLEX using the IBM ILOG Concert API. We used default CPLEX settings, except we increased the maximum allowed memory usage to the total amount of free physical memory. Since the proposed heuristic is probabilistic, we applied it to each of the test cases 10 times. The pseudorandom number generator we used was the MT19937 Mersenne Twister [12]. For the weighted objective function, we used $\alpha=\beta=1$. We also applied the GA matheuristic to each of the test cases 10 times for the same amount of time that the LR-based heuristic used on average.

Table 1 shows the results of the computational experiments. The tables shows the test case (Case), the number of students (Std), the number of classes (Cls), the number of student types (Typ), the number of variables in the QP (Vars), the minimum (tMin), average ( $\mathrm{tAvg}$ ), and maximum (tMax) solution time for the LR-based heuristic, the minimum (lrMin) and maximum (lrMax) objective

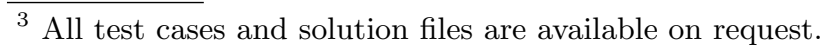


value obtain by applying the LR-based heuristic, and the minimum (gaMin) and maximum (gaMax) objective value obtain by applying the GA matheuristic. Since the two approaches tested are quite different, time is reported in CPU time. It is clear from the results that the LR-based heuristic outperformed the GA matheuristic.

In Figure 1, the horizontal axis depicts the 90 test cases. The vertical axis gives the time taken, in seconds. The graph shows the range of solution times across the 10 runs of the LR-based heuristic. The solid line shows the average time across the 10 runs of the heuristic. The largest value was about 40 minutes, however the overall average was only about 272 seconds. In contrast, the attempts to obtain exact solutions by solving the corresponding quadratic programming problem using CPLEX failed in most cases given a six hour limit.

LR-based Heuristic Solution Times

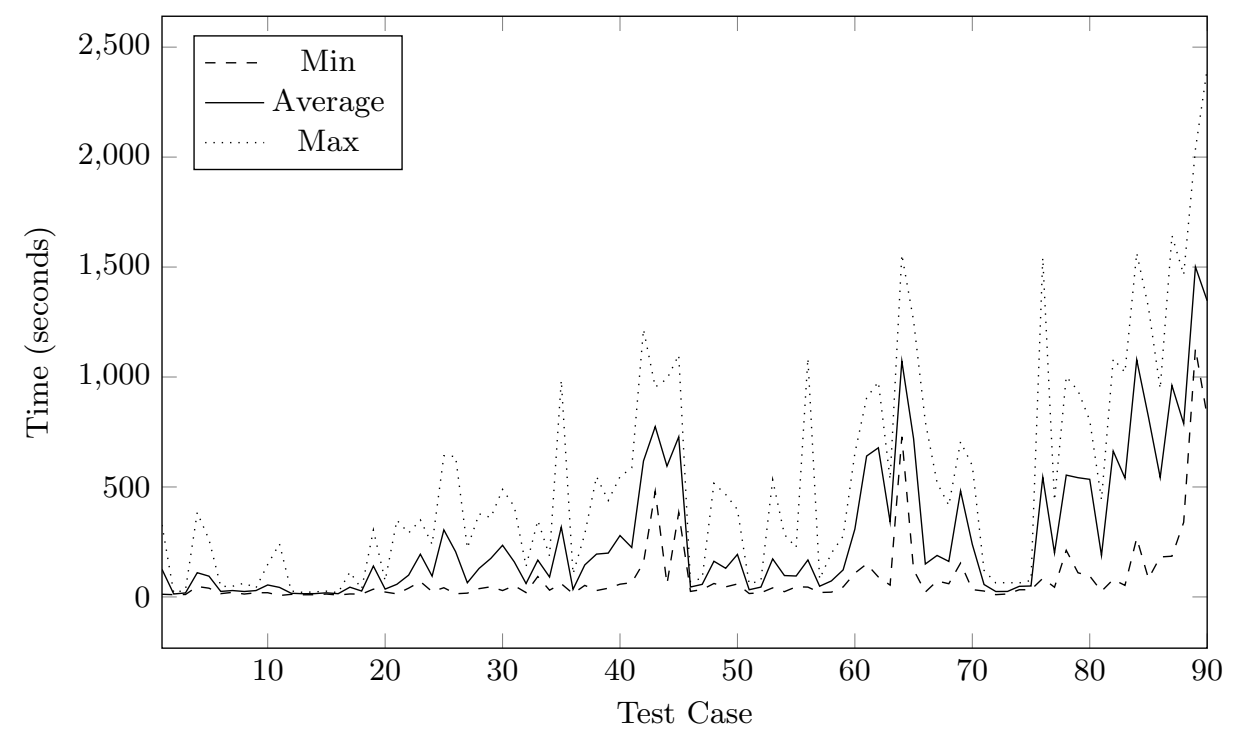

Fig. 1. The time taken for the LR-based heuristic to produce a solution.

\section{Conclusions}

In this paper we presented a heuristic solution approach, based on the Lagrangian relaxation, for the problem of assigning students to classes. This problem arises in large organisations that require training and retraining of staff. The objective function reflects the preference of assigning certain groups of students to the same class, which often occurs in practice. The proposed heuristic was tested by computational experiments on a number of randomly generated test cases, 


\begin{tabular}{|c|c|c|c|c|c|c|c|c|c|c|c|}
\hline ase & $\mathrm{td}$ & Cls & Typ & Vars & tMin & tAvg & tMax & lrMin & $\operatorname{lrMax}$ & gaMin & gaMax \\
\hline 1 & 300 & 56 & 3 & 62776 & 12.1 & 125.4 & 327.1 & 1700.5 & 14101.5 & 7979 & 13904.5 \\
\hline 2 & 00 & 56 & 3 & & 0.3 & 3.4 & 18.4 & 483.5 & 3009 & 20452 & 6405 \\
\hline 4 & 0 & 38 & 3 & & 7.5 & 10.1 & 83.8 & 26 & 277 & 8.5 & 0827. \\
\hline 5 & 00 & 98 & 3 & 58 & 39.7 & 94.4 & 61.1 & 1328.5 & 012 & 47.5 & 218 \\
\hline 7 & 91 & 66 & 4 & & 20.8 & 29 & 8.4 & 1883.5 & 2405 & 13872 & \\
\hline 8 & 91 & 78 & 4 & 736 & 13.1 & 24.7 & 62.5 & 2160 & 2739.5 & & 22527.5 \\
\hline 10 & 91 & 98 & 4 & 108976 & 19.2 & 54.4 & 147.6 & 1904 & 6210 & 50.5 & 22959.5 \\
\hline 11 & $\overline{06}$ & 56 & 4 & & 6.5 & 43.4 & 241.5 & 1437 & 17346.5 & 5930.5 & 10101 \\
\hline 13 & 206 & 78 & 4 & 80106 & 9.8 & 14.6 & 20.5 & 1443.5 & \begin{tabular}{|c|}
3482 \\
\end{tabular} & 12167 & 16141 \\
\hline 14 & $\overline{206}$ & 38 & 4 & 90376 & 10 & 15 & 23 & 1236 & 12347 & 13018.5 & 16949 \\
\hline 16 & $\overline{300}$ & 56 & 6 & 62776 & 8.6 & 14.7 & 19.4 & 1597 & 7193.5 & 21518.5 & 31016 \\
\hline 17 & 300 & 66 & 6 & 73986 & 13.5 & 44.3 & 113.4 & 1574.5 & 3314 & 11135.5 & 21437 \\
\hline 19 & 300 & 88 & 6 & 98648 & 35.4 & 140.5 & 303.9 & 1341 & 3251 & 10727 & 18855 \\
\hline 20 & $\overline{300}$ & 98 & 6 & 109858 & 22.3 & 35.6 & 73.2 & 1136.5 & 3634.5 & 20977 & 25647 \\
\hline 22 & 400 & 66 & 5 & 80586 & 39.9 & 99 & 290.6 & 2181 & 5061 & 13182.5 & 28905 \\
\hline 23 & 400 & 78 & 5 & 5238 & 69.8 & 194.1 & 349.9 & 2078 & 2665.5 & 11723.5 & 25204 \\
\hline 25 & 400 & 98 & 5 & 119658 & 41.8 & 04.8 & 642.1 & 1665 & 7581 & 11361.5 & 26601 \\
\hline 26 & 500 & 56 & 6 & 73976 & 14.4 & 05.8 & 638.5 & 3004.5 & 16429 & 16148 & 38892.5 \\
\hline 28 & 500 & 78 & 6 & 103 & 37.6 & 29.6 & 377.8 & 3117 & 4130.5 & & 43548. \\
\hline 29 & 500 & 88 & 6 & 116248 & 46.1 & 75.2 & 361.3 & 2389.5 & 7289 & 23430 & 45908. \\
\hline 31 & 132 & 56 & 7 & 53368 & 51.2 & 59.1 & 411 & 1454.5 & 1654.5 & 324.5 & 7100 \\
\hline 32 & 132 & 6 & 7 & & 19.7 & 1.1 & 38.6 & 1383 & 549.5 & 5418 & 6608 \\
\hline 34 & 132 & 8 & 7 & & 30.7 & 0.7 & 86.4 & 1518. & 106 & 4081 & 6520 \\
\hline 35 & 32 & 8 & 7 & & 60 & 7.4 & 85.1 & 1292 & 890 & 7.5 & 6680 \\
\hline 37 & 400 & 66 & 5 & & 52.5 & 5.4 & 285.9 & 1889 & 3319.5 & 11636.5 & 24165 \\
\hline 38 & 100 & 78 & 5 & & 29 & 4.7 & 547.5 & 2262 & 5.5 & 23962 & 26256.5 \\
\hline 40 & 100 & 98 & 5 & & 5 & 279.1 & 546.7 & 1639 & .5 & 11082.5 & 25742 \\
\hline 41 & 500 & 56 & 8 & & 64.9 & 224.9 & 587 & 3786.5 & 11623 & 14888.5 & 37871.5 \\
\hline 43 & 00 & 78 & 8 & 03038 & 480.3 & 774.3 & 954.3 & 3095.5 & 7006.5 & 94 & 29130.5 \\
\hline 44 & 00 & 8 & 8 & 16248 & 58.3 & 94.7 & 991.5 & 2574 & 3868.5 & 10993.5 & 32387.5 \\
\hline 46 & 300 & 56 & 6 & & 25.1 & 45.1 & 69.6 & 2274.5 & 18798 & & 21706.5 \\
\hline 47 & 300 & 6 & 6 & & 33.6 & 7.2 & 85.3 & 2183.5 & 8768 & & 19975.5 \\
\hline 49 & 300 & & 6 & & 45.7 & 130.2 & 466 & & 7697.5 & 481.5 & 19685 \\
\hline 50 & 300 & 98 & 6 & & 58.9 & 93.2 & 397.4 & 1778.5 & 3082 & 32 & 18988 \\
\hline 52 & 168 & 6 & 6 & & 19 & 4.7 & 74.1 & 1637 & 2614 & 321.5 & 8415 \\
\hline 53 & 168 & 8 & 6 & & 41.2 & 172.9 & 532.7 & 605.5 & 2674 & & 318 \\
\hline 55 & 168 & 98 & 6 & & & 95.1 & 224.9 & 1404.5 & 050.5 & 505.5 & 9456 \\
\hline 56 & 500 & 56 & 5 & & 44.7 & 168 & 1084.7 & 2825 & 13742 & 15216.5 & 40438 \\
\hline 58 & 500 & 78 & 5 & 038 & 21.7 & .7 & 199.1 & 2550.5 & 3835 & 2732.5 & 44762.5 \\
\hline 59 & 500 & 38 & 5 & 248 & 45.3 & 22.9 & 276.3 & 2465.5 & 13736.5 & \begin{tabular}{|l|}
36664.5 \\
\end{tabular} & 47615.5 \\
\hline 61 & 300 & 56 & 7 & & 49.9 & 40.6 & 904.9 & 2286 & 3305.5 & 7636.5 & 12557 \\
\hline 62 & 300 & 66 & 7 & & 90.6 & 78.1 & 978 & 2312.5 & 2786.5 & 10760.5 & 14360 \\
\hline 64 & 300 & 88 & 7 & & 28 & 1071.9 & 1556.5 & 2215 & 2666.5 & 25 & 12500.5 \\
\hline 65 & 300 & 98 & 7 & & 123.3 & & 1256.1 & 1999.5 & 4367.5 & 9902.5 & 16164 \\
\hline 67 & $\overline{00}$ & 66 & 8 & & 2.6 & & 9.3 & 210 & 74.5 & 16810.5 & 24442.5 \\
\hline 68 & 00 & 78 & 8 & & 60 & & & & & & 2661 \\
\hline 70 & 400 & 98 & 8 & & 33.3 & 9.1 & 597.3 & 1584.5 & \begin{tabular}{|l|}
2056.5 \\
\end{tabular} & 11480.5 & \begin{tabular}{|l|}
27258 \\
\end{tabular} \\
\hline 71 & 132 & 56 & 7 & & & 56.4 & 120.8 & 1237 & 95 & 5303 & 7409.5 \\
\hline 73 & 32 & 78 & 7 & & .4 & 25 & 65 & 1230.5 & \begin{tabular}{|l|}
1910.5 \\
\end{tabular} & 7.5 & 7684.5 \\
\hline 74 & 132 & 38 & 7 & & & 3.2 & 3.6 & 1296 & 1665 & 5611.5 & 6721 \\
\hline 76 & 300 & 56 & 9 & & .4 & 4.1 & 542.1 & 2189 & 13773 & 96 & 11942.5 \\
\hline 77 & 300 & 66 & 9 & & .1 & 0.3 & 438.4 & 1982.5 & 2979 & \begin{tabular}{|l|}
10571.5 \\
\end{tabular} & 16390 \\
\hline 79 & 300 & 88 & 9 & 48 & 109.9 & 42.2 & 938 & 2254 & 3810.5 & 7113 & 15953.5 \\
\hline 80 & 300 & 98 & 9 & 558 & 95 & 34.7 & 02.9 & 1958.5 & 3169.5 & 8544 & 12726 \\
\hline 82 & 400 & 66 & 11 & & 79.5 & 33.1 & 82.2 & 2577 & 6110 & 10912 & 16338 \\
\hline 83 & 400 & 78 & 11 & & 52.5 & 40.4 & 1.6 & 2681.5 & 3984 & 10771.5 & 23656. \\
\hline 84 & 400 & 88 & 11 & & 267.5 & & .9 & 2512.5 & \begin{tabular}{|l|}
5764.5 \\
\end{tabular} & & 14386.5 \\
\hline 85 & 400 & 98 & 11 & 658 & 83.4 & & & 2404.5 & 5749 & 10067.5 & 23096 \\
\hline 86 & 500 & 56 & 11 & & & & & 3735 & 17272.5 & \begin{tabular}{|l|}
16703 \\
\end{tabular} & 17796 \\
\hline 87 & 500 & 66 & 11 & & & 961.3 & 1643.4 & 3624 & 8932.5 & 14413.5 & 20936 \\
\hline 88 & 500 & 78 & 11 & 103038 & 339.4 & & 1465 & 3709.5 & 7593 & 13059.5 & 30584. \\
\hline 89 & 500 & 88 & 11 & 116248 & 1130.2 & 1501 & 2035.3 & 3185 & 16049.5 & 10998 & 21648.5 \\
\hline 90 & $\overline{500}$ & 98 & 11 & 129458 & 821.2 & 347.1 & 2401 & 2724.5 & 9361 & 10298.5 & 29047 \\
\hline
\end{tabular}

Table 1. The results of the computational experiments for many of the test cases. 
based on data supplied by Ausgrid. The proposed heuristic was able to provide solutions to all test cases in a practically acceptable time. In contrast, the straight forward quadratic programming based approach failed in most cases with a time limit of six hours. In both cases, CPLEX was used as the solver. The Lagrangian relaxation-based heuristic was also compared with a specifically designed matheuristic based on Genetic Algorithms. This comparison indicated the superiority of the Lagrangian relaxation-based heuristic. The integer programming components of the matheuristic were also solved with CPLEX.

The proposed Lagrangian relaxation-based heuristic includes a number of parameters, and future research can be focussed on an investigation of the influence of these parameters on the performance of the entire procedure.

\section{References}

1. Caprara, A., Pisinger, D., Toth, P.: Exact solution of the quadratic knapsack problem. INFORMS Journal on Computing 11(2), 125-137 (1999)

2. Chen, Y., Hao, J.K.: Iterated responsive threshold search for the quadratic multiple knapsack problem. Ann Oper Res 226, 101-131 (2015)

3. Chopra, S., Rao, M.R.: The partition problem. Mathematical Programming 59(13), 87-115 (1993)

4. Dahlhaus, E., Johnson, D.S., Papadimitriou, C.H., Seymour, P.D., Yannakakis, M.: The complexity of multiway cuts. In: Proceedings of the twenty-fourth annual ACM symposium on Theory of computing. pp. 241-251. ACM (1992)

5. Fisher, M.L.: The lagrangian relaxation method for solving integer programming problems. Management science 50(12_supplement), 1861-1871 (2004)

6. García-Martínez, C., Rodriguez, F., Lozano, M.: Tabu-enhanced iterated greedy algorithm: A case study in the quadratic multiple knapsack problem. European Journal of Operational Research 232, 454-463 (2014)

7. Garey, M.R., Johnson, D.S.: Computers and intractability: a guide to npcompleteness (1979)

8. Guignard, M.: Lagrangean relaxation. Top 11(2), 151-200 (2003)

9. Hiley, A., Julstrom, B.A.: The quadratic multiple knapsack problem and three heuristic approaches to it. In: Proceedings of the 8th annual conference on Genetic and evolutionary computation. pp. 547-552. ACM (2006)

10. Johnson, E.L., Mehrotra, A., Nemhauser, G.L.: Min-cut clustering. Mathematical programming 62(1-3), 133-151 (1993)

11. Julstrom, B.A.: Greedy, genetic, and greedy genetic algorithms for the quadratic knapsack problem. In: Proceedings of the 7th annual conference on Genetic and evolutionary computation. pp. 607-614. ACM (2005)

12. Matsumoto, M., Nishimura, T.: Mersenne twister: a 623-dimensionally equidistributed uniform pseudo-random number generator. ACM Transactions on Modeling and Computer Simulation (TOMACS) 8(1), 3-30 (1998)

13. Saraç, T., Sipahioglu, A.: A genetic algorithm for the quadratic multiple knapsack problem. In: Advances in Brain, Vision, and Artificial Intelligence, pp. 490-498. Springer (2007)

14. Singh, A., Baghel, A.S.: A new grouping genetic algorithm for the quadratic multiple knapsack problem. In: Evolutionary Computation in Combinatorial Optimization, pp. 210-218. Springer (2007) 